\title{
Risk genes, metabolic syndrome and eye tracking deficits in psychiatric diseases
}

\author{
Andrea Schmitt • Peter Falkai
}

Published online: 3 March 2013

(c) Springer-Verlag Berlin Heidelberg 2013

Stress is a common environmental factor playing a major role in psychiatric diseases. Since the mineralocorticoid receptor is dominantly involved in the initial triggering of stress response, Kortmann et al. [1] investigated the possible role of its encoding gene $\mathrm{NR} 3 \mathrm{C} 2$, a candidate for psychiatric disorders, in the symptom dimensions and susceptibility to persistent ADHD. The Val allele of the MRI180 V polymorphism (rs5522) evidentially increases the risk of depression, attention and cognitive deficits. They accordingly compared genotype and allele frequencies to symptom dimensions each with samples of adult ADHD patients and controls. The group found carriers of the Val allele to present higher inattention, hyperactivity/ impulsivity and impairment scores, whereas patients and controls showed now differences in genotype and allele frequencies, thus confirming a possible link between genetic variations in the HPA axis and inattention and hyperactivity measures. Hoare et al. [2] again hypothesized the gene Apolipoprotein E (ApoE) to be relevant to the individual variation in acquiring the serious neuropsychiatric disorder HIV-associated dementia (HAD). They investigated the relationship of ApoE genotype to neuropsychological function plus white matter integrity of the corpus callosum via DTI in a ROI of 45 HIV-positive subjects with clade C HIV. Comparing subjects with at least one $\varepsilon 4$ allele to subjects with no $\varepsilon 4$ allele showed significantly decreased immediate and delayed recall plus significantly decreased fractional anisotropy in the corpus callosum for the first group. Thus, the authors suppose the

A. Schmitt $(\bowtie) \cdot$ P. Falkai

Department of Psychiatry and Psychotherapy,

Ludwig-Maximilians-University Munich,

Nußbaumstr. 7, 80336 Munich, Germany

e-mail: Andrea.Schmitt@med.uni-muenchen.de $\varepsilon 4$ allelic variant of ApoE to be associated with memory impairment and white matter damage of the corpus callosum in HIV patients.

Social exclusion is processed in the anterior cingulate cortex (ACC) for which reason Frischknecht et al. [3] assumed a correlation of chronic social exclusion with altered neural plasticity reflected by neurometabolic alterations in the ACC. To that effect, a highly stigmatized group of 15 heroin-addicted patients during substitution therapy rated a stigmatization questionnaire, while in parallel neurometabolic markers in the ACC were measured with ${ }^{1} \mathrm{H}$ MR spectroscopy. In patients stating high discrimination, there was a negative correlation between this experience and $N$-acetylaspartate (NAA) pointing to attenuated neuron functioning in the anterior cingulate cortex. In the same group, an association with anxiety mediated by NAA was found. In spite of missing evidence, the authors assume that the relation of NAA in the ACC and discrimination might indicate a malfunction of the neural system involved in cognitive control over emotionally relevant social stimuli in these patients.

Kahl et al. [4] explored the prevalence of the metabolic syndrome (MetS) in 135 patients fulfilling DSM-IV criteria for borderline personality disorder (BPD) compared to 1,009 control subjects from primary care and found a more than doubled age-standardized prevalence of MetS in BPD patients. As to individual MetS criteria, in both genders, hyperglycemia was significantly more prevalent. For female BPD patients, the same applied to abdominal obesity and hypertriglyceridemia. Overall, an increased rate of MetS within BPD patients was associated with significant higher BMI, age, treatment with second-generation antipsychotics, dysthymia, panic disorder, benzodiazepine dependency and binge eating disorder. Since MetS is a known risk factor for developing type- 2 diabetes and 
coronary artery disease, the authors claim careful cardiometabolic monitoring and screening for physical health conditions among BPD patients.

Because of feasible impaired volitional saccade control in patients with obsessive-compulsive disorder (OCD), Kloft et al. [5] hypothesized this deviance to be a cognitive endophenotype. They compared 22 unaffected first-degree relatives of OCD patients as genetic risk carriers to 22 unmedicated OCD patients and 22 healthy controls without such family history. Performing two types of volitional saccade tasks measuring response selection or only response initiation plus visually guided saccades as control condition, the patient and relatives groups alike were significantly slower in volitional response selection than healthy controls. The authors thus possibly have found first evidence for a candidate endophenotype for OCD. Eye tracking deficits occurring in schizophrenia (SZ) have been ascribed to alterations in sensorimotor processing and predictive mechanisms. In this respect, Sprenger et al. [6] conducted a study with $20 \mathrm{SZ}$ patients and 20 healthy controls assessed on blocks of predictably moving visual targets at constant speeds of 10,15 or $30 \%$ which were blanked off for different periods during the ongoing pursuit movement. Besides residual eye velocity during blanking intervals, they measured persistence of predictive signals via eye deceleration after extinction of the visual target, which was slower in patients than controls, thus bespeaking rather greater than diminished utilization of predictive mechanisms for pursuit in SZ. Patients additionally showed no impairment in residual gain which indicates a basic integrity of internal predictive models. However, reduced pursuit velocity gain in SZ patients in all conditions with visible targets suggests primarily impaired sensorimotor transformation of the retinal error signal needed for maintaining accurate visually driven pursuit. The authors conclude that rather disruption in extrastriate and sensorimotor systems underlies this SZ endophenotype and less frontostriatal predictive mechanisms.

Sanders et al. [7] seized the suggestion of impaired perceptual inference being at the core of positive SZ symptoms via testing the strength of apparent motion (AM) in 34 patients with paranoid SZ compared to 34 healthy controls. AM is a visual illusion triggering the impression of seeing one object moving back and forth when two spatially separate objects are alternately flashed. Patients had a lower probability of motion perception and thus showed to be less susceptible to illusion while the probability of AM perception, again, was inversely related to delusional conviction. Thus, SZ might be associated with reduced susceptibility to visual fallacies commonly relying on perceptual inference. With 24 SZ patients and 24 matched controls performing auditory and visual oddball tasks, Neuhaus et al. [8] show how the thus obtained event-related potential (ERP) data can be applied to classify a pathological state in single subjects without knowing their diagnoses. They consider this method of machine learning algorithms to have the potential for the identification of potential biomarkers. Fond et al. [9] tested in $63 \mathrm{SZ}$ patients and 67 healthy controls, whether patients display impairments in profitable decision-making, one key concept to understand some behavioral disturbances in SZ, on experimental designs. The study groups undertook the Iowa gambling task (IGT) plus the game of dice task (GDT), and additionally, clinical variables were evaluated with questionnaires the same day and pharmacological treatments encompassed. SZ patients showed impaired performances on both decision-making tasks, but no correlation between these results and clinical variables could be found. Hence, SZ patients display impairments in both decision-making under ambiguity and under risk. Focussing on patient-parent relationships as a significant environmental factor in the long-term course of SZ, Choi et al. [10] used fMRI to study neural representation of parents in $14 \mathrm{SZ}$ outpatients and 15 healthy controls. Patients reporting a better communicative fluency with one's mother demonstrated greater neural activation in the superior temporal sulcus and the parahippocampal gyrus for parental stimuli. Contemplating their mothers, activities of SZ patients in these regions were associated with a level of negative symptoms of affective ambivalence. Thus, the authors conclude that parental cues are processed in a more socially driven manner and emphasize the importance of prudent family interventions in the psychiatric rehabilitation of SZ patients.

\section{References}

1. Kortmann GL, Contini V, Bertuzzi GP, Mota NR, Rovaris DL, Paixão-Côrtes VR, Bau CHD (2012) The role of a mineralocorticoid receptor gene functional polymorphism in the symptom dimensions of persistent ADHD. Eur Arch Psychiatry Clin Neurosci. doi:10.1007/s00406-012-0321-z

2. Hoare J, Westgarth-Taylor J, Fouche JP, Combrinck M, Spottiswoode B, Stein DJ, Joska JA (2012) Relationship between apolipoprotein E4 genotype and white matter integrity in HIVpositive young adults in South Africa. Eur Arch Psychiatry Clin Neurosci. doi:10.1007/s00406-012-0341-8

3. Frischknecht U, Hermann D, Heinrich M, Hoerst M, Weber-Fahr W, Vollstädt-Klein S, Ende G (2012) Experience of social discrimination correlates with neurometabolism: a pilot study in heroin addicts. Eur Arch Psychiatry Clin Neurosci. doi: 10.1007/s00406-012-0319-6

4. Kahl KG, Greggersen W, Schweiger U, Cordes J, Correll CU, Frieling H, Moebus S (2012) Prevalence of the metabolic syndrome in patients with borderline personality disorder: results from a cross-sectional study. Eur Arch Psychiatry Clin Neurosci. doi:10.1007/s00406-012-0339-2

5. Kloft L, Reuter B, Riesel A, Kathmann N (2012). Impaired volitional saccade control: first evidence for a new candidate 
endophenotype in obsessive-compulsive disorder. Eur Arch Psychiatry Clin Neurosci. doi:10.1007/s00406-012-0331-x

6. Sprenger A, Trillenberg P, Nagel M, Sweeney JA, Lencer R (2012) Enhanced top-down control during pursuit eye tracking in schizophrenia. Eur Arch Psychiatry Clin Neurosci. doi:10.1007/ s00406-012-0332-9

7. Sanders LLO, de Millas W, Heinz A, Kathmann N, Sterzer P (2012) Apparent motion perception in patients with paranoid schizophrenia. Eur Arch Psychiatry Clin Neurosci. doi:10.1007/ s00406-012-0344-5

8. Neuhaus AH, Popescu FC, Bates JA, Goldberg TE, Malhotra AK (2012). Single-subject classification of schizophrenia using event- related potentials obtained during auditory and visual oddball paradigms. Eur Arch Psychiatry Clin Neurosci. doi:10.1007/ s00406-012-0326-7

9. Fond G, Bayard S, Capdevielle D, Del-Monte J, Mimoun N, Macgregor A, Raffard S (2012) A further evaluation of decisionmaking under risk and under ambiguity in schizophrenia. Eur Arch Psychiatry Clin Neurosci. doi:10.1007/s00406-012-0330-y

10. Choi SH, Lee SH, Park HJ, Chun JW, Kang JI, Kim JJ (2012) Perceived patient-parent relationships and neural representation of parents in schizophrenia. Eur Arch Psychiatry Clin Neurosci. doi:10.1007/s00406-012-0334-7 